\title{
Characterization of Microstructures using the Chord Length Transformation
}

\author{
K. Sandau
}

Faculty of Mathematics and Science, University of Applied Sciences Darmstadt, Schöfferstraße 3, D-64295 Darmstadt, Germany

Many tools of image analysis are based on methods used in quantitative microscopy and are applied since a long time. But nowadays digital image analysis offers tools which need such a huge effort, that they never would be applied without a machine. The recently developed chord length transformation (CLT) is such a tool which helps to segment structures and which also helps to find suitable features for classifying structures [1]. This contribution presents the CLT and applies it at different levels of quantitative image analysis.

The CLT assigns to every pixel in the foreground of a binary image and to each angle (of a discrete set of angles) the length of the chord. This length is measured along a line through the pixel point having a slope derived from this angle. The length is the length of the connecting chord determined by the intersection of the line with the foreground. For each fixed angle the resulting data set can be depicted as a gray value image where the gray values are proportional to the chord lengths. Figure 1.a shows a binary image and Figure 1.b shows the image of chord lengths for the angle $\pi / 6$. From the whole data set (all angles) statistical information can be derived. So maximal and minimal chord lengths can be assigned to each pixel. A visualization of maximal chord lengths is given in Figure 1.c.

The local information can be used for the segmenting process as is shown in the first example, where the CLT was applied to a binary image showing lamellas of graphite in gray cast iron. A specimen, grinded and etched, was regarded using a light microscope equipped with a lens of magnification 10. Then graphite can be seen embedded in the steel phase. One typical arrangement of the graphite is an arrangement in lamellas (Figure 1.a). For further measurements connected lamellas should be segmented in line shaped parts. The segmentation has been done here using the CLT as a 3D data structure and thresholding local adaptively using the local information about maximal and minimal chord lengths. Afterwards each segment in $3 \mathrm{D}$ can be projected to the $2 \mathrm{D}$ image. In this way crossing lamellas can be measured one by one. Figure. 1.d illustrates this (the arrow in the lower left corner points at a configuration, which is segmented into three parts two are vertical and one is longitudinal arranged).

A second example uses the information along the angles to get an angle distribution and to segment the image with respect to the local main directions. This is demonstrated here for a microscopical image of a perlitic microstructure (Fig. 2.a). Obviously one observes several main areas of homogeneous direction. These areas are characterized by the direction of the maximal chord length in the foreground and in the background image. Figure 2.c shows the result of the segmentation process. The smoothed regions partition the image in the main areas as expected by the human eye.

The data set given by the CLT obviously allows to estimate the chord length distribution. This distribution is studied in stochastic geometry [2-3], often in the context of stochastic germ-grain models. If the germ-grain model is especially a Boolean model one knows the distribution function for the chord lengths in the background. Based on this knowledge a parameter can be developed, describing a kind of distance to 'Boolean model'. As a final example this parameter is applied here to 
the lamellas of graphite in gray cast iron as described above. Following the DIN EN ISO 945 or the counterpart ASTM A 247-47 respectively, five types of arrangement of the lamellas are distinguished. To demonstrate the method, the parameter is calculated for arrangements of type A and type $\mathrm{E}$ and the result shows a good discrimination property. The parameter indicates that type A graphite is closer to the Boolean model than type E, which coincides with other results [4].

\section{References:}

[1] K. Sandau and J. Ohser, Acta Stereol. (2005, submitted).

[2] J. Ohser and F. Mücklich, Statistical Analysis of Microstructures in Materials Science. J. Wiley \& Sons, Chichester, 2000.

[3] D. Stoyan et al., Stochastic Geometry and Applications. 2nd Edition. J. Wiley \& Sons, Chichester, 1995.

[4] O. Liesenberg and J. Ohser, Gießereitechnik 29 (1983) 106.
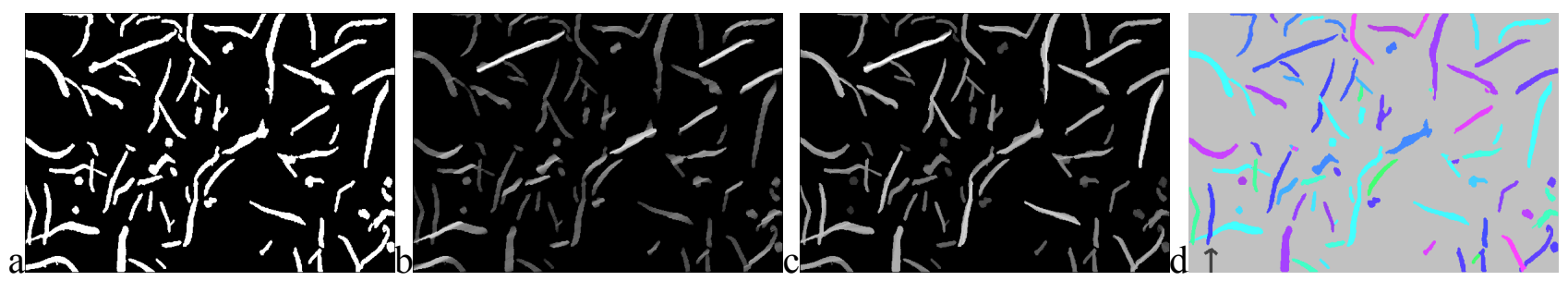

Fig. 1. Lamellar graphite in gray cast iron. a. binary image. b. The chord lengths of the chords in the direction $30^{\circ}$, depicted by the gray values. c. The maximal chord length at each pixel. $\mathrm{d}$. The segmented image derived from 1.a. The white arrow in the lower left corner in particular shows a segmentation of one connected object to three line shaped objects.
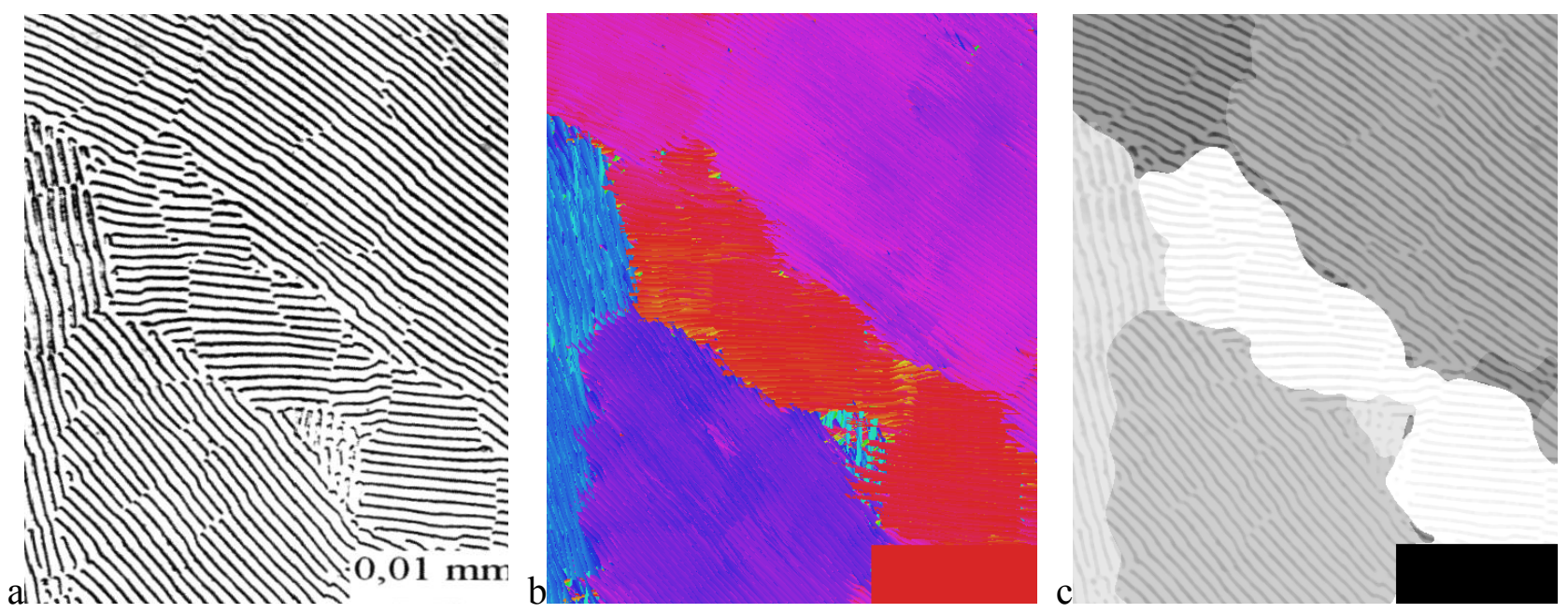

Fig. 2. Perlitic microstructure. a. The gray value image after some preprocessing routines. b. A color coded image of the directions. A color is assigned to each direction following the HLS colorspace (red $=0^{\circ}$, green $=60^{\circ}$, blue $\left.=120^{\circ}\right)$. c. The segmentation with respect to the directions has been overlayed to the original image. 